\title{
Study on Metrics Model for Energy Efficiency in Data Centers
}

\author{
Changgeng $\mathrm{Yu}^{1, \mathrm{a}}$ and Liping Lai ${ }^{2, \mathrm{~b}}$ \\ ${ }^{1}$ School of Mechanical and Electronic Engineering, Hezhou University, No.18, Xihuan Road, Hezhou, \\ China \\ ayuchanggen66@163.com, 'lailiping66@163.com
}

Keywords: Data centers; Energy efficiency; Metrics; PUE; Energy efficiency evaluation

\begin{abstract}
With the high costs of power, energy efficiency has rapidly become a critical consideration issue when evaluating data centers. Energy efficiency metric can be used by managers to measure and maintain the implementation of cost saving and $\mathrm{CO}_{2}$ emissions in data centers. In this study, a metric model for energy efficiency in data centers is created to provide a reference. Three of the most important data center metrics, PUE, CUE and DCeP, are examined closely. The paper provides a good comprehension of multiple approaches and makes recommendations for a platform metric that can be further developed and adopted as a standard.
\end{abstract}

\section{Introduction}

Energy consumption in data centers is increasing year by year with the growth in data centers market. In order to mitigate global warming, it's an urgent task to reduce power consumption in data centers. Generally, ICT equipment (e.g., servers, routers, switches, and storage units) and facility equipment (e.g., power delivery components, heating ventilation and air conditioning (HVAC) system components) account for a large percentage of energy consumption in data centers. Thus, power-saving measures for these components are necessary to reduce overall power consumption.

To strengthen the energy efficiency evaluation of enterprises, improve the efficiency of data center energy has become the priority of data center monitoring system. Global industry and academia are widely focus on problems of energy efficiency evaluation, for example, the U.S. Department of Energy (DOE) was built in an organization, in order to improve the energy efficiency, as the primary goal of Industrial Assessment Centers, IAC[1-3]. The center, relying on 29 colleges, universities, and industrial sectors in the United States, has set up a file in more than 15000 U.S. companies implement Suggestions on the industrial energy audit and evaluation of projects; Such as Green Grid organization (Green Grid) was founded in 2007 [4], the combined company, government, industry, research and try to provide the best data center energy saving methods, assessment methods and techniques; Founded in the same year, Green500 group, the group in June and December each year respectively issued to MegaFlops/W (i.e., per watt power can complete one million floating point calculations per second) as an index, efficiency of 500 sets of the world's fastest supercomputer, sorting; EPA (in January 2010, the United States environmental Protection of the Environment Protection, EPA) and the Department of Energy, DOE launched the national data center Energy efficiency information project, ASHRAE, The Green Grid, such as eight professional association to a data center in Washington, data center Energy efficiency evaluation standard meeting, hope to make joint data center Energy efficiency evaluation standard, in order to improve data center Energy efficiency and reduce Energy consumption [5].

To sum up, the data center energy efficiency assessment has rise at home and abroad. However, existing research is scattered and due to the data center have the characteristics of wide range, so manyproblems in the evaluation of the efficiency of data center still to be solved.

\section{Metric Model for Energy Efficiency in Data Centers}

Energy efficiency assessment metric in Data center is based on efficiency evaluation technical, is quantitative standard by measure of data center energy efficiency, and can reflect the energy utilization 
in the process of data center operation, for the data center provide important basis for design, process and management, and provide a reference basis to be compared to the energy efficiency of different data center.

Evaluation metrics model for energy efficiency in data center is shown in Fig. 1. Data center energy efficiency metrics mainly components from the energy consumption (IT equipment and infrastructure energy consumption), $\mathrm{CO}_{2}$ emissions and performance evaluation, etc.

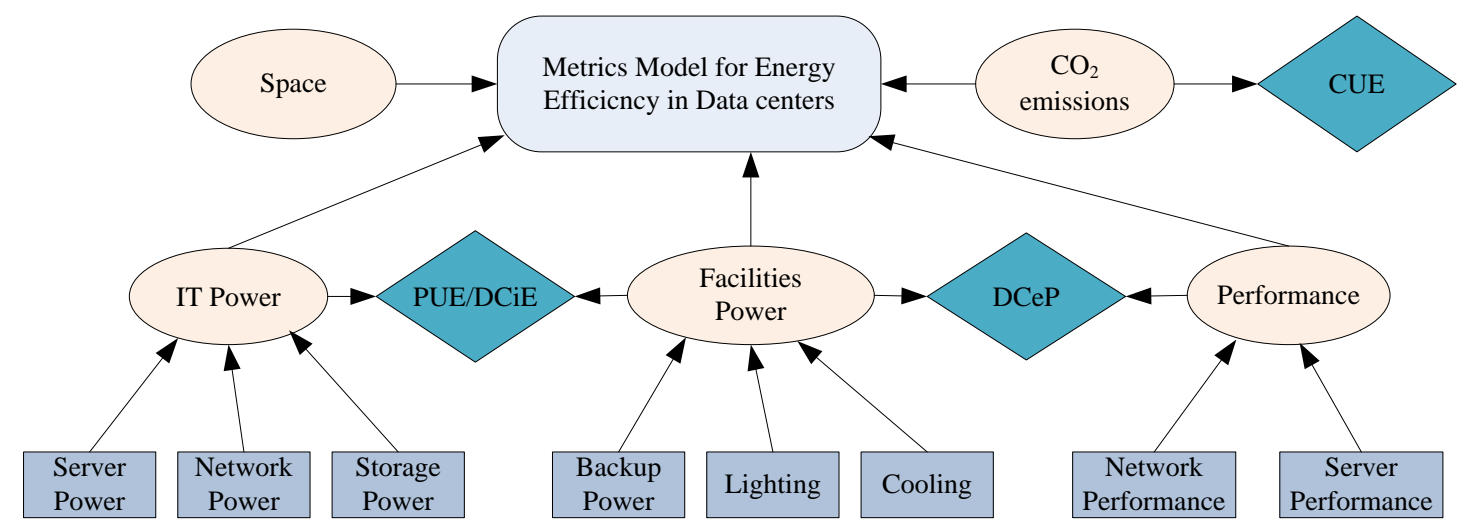

Figure 1. Metrics Model for Energy Efficiency in Data Centers

(1). Power Usage Effectiveness (PUE). While there are many efficiency metrics, the preferred data center efficiency metric is Power Usage Effectiveness, PUE. The PUE metric, developed by the Green Grid [6], is defined as the ratio of the total facility power coming into a data center to that consumed by the IT equipment. PUE can be calculated by:

$$
\mathrm{PUE}=\frac{\text { Total Facility Power }}{\text { IT Equipment Power }}
$$

While PUE is one of the most commonly used efficiency metrics, it does not reflect all efficiency characteristics of a data center. In particular, PUE may not adequately capture improvements in the energy efficiency of IT equipment, because reductions in energy consumed by IT equipment are reflected both in the numerator and the denominator in the PUE formula. Some metrics that highlight other aspects of data center energy efficiency include Corporate Average Data Center Efficiency (CADE), Data Center Infrastructure Efficiency (DCiE), Data Center energy Productivity (DCeP), and Energy Reuse Effectiveness (ERE).

The PUE metric shows that the relationship between the IT equipment energy consumed and the total energy consumed of data center. According to the data center energy consumption structure can be concluded that the main factors influencing the PUE for IT equipment energy consumed, the refrigeration system energy consumed and other energy consumed that is basically a constant value.

For example, suppose that IT equipment energy consumed of $x \mathrm{kw}$, refrigeration system energy consumed to $y \mathrm{kw}$, and other energy consumed for $Q \mathrm{kw}$, PUE of the function $f(x)$ is expressed as:

$$
f(x)=(x+y+Q) / x
$$

(2). Data Center Infrastructure Efficiency (DCiE). This metric is just the reciprocal of PUE. Thus, it gives the efficiency of the power brought inside the data center in being utilized by the IT equipment. It can be calculated by [6]:

$$
\mathrm{DCiE}=\frac{1}{\text { PUE }}=\frac{\text { IT Equipment Power }}{\text { Total Facility Power }} \times 100 \%
$$

(3). Carbon Usage Effectiveness (CUE). A new sustainability metric, known as Carbon Usage Effectiveness (CUE), complements energy efficiency metric, in particular the PUE metric, to provide insight into another dimension of data center environmental performance. 
CUE [7], also developed by TCG, is a metric that enables an assessment of the total Greenhouse Gas emission of data center, relative to its IT energy consumption. CUE is computed as the total carbon dioxide emission equivalents $\left(\mathrm{Co}_{2} \mathrm{eq}\right)$ form the energy consumption of the facility divided by the total IT energy consumption.

$\mathrm{CUE}=$ Total $\mathrm{Co}_{2}$ emissions (kg Co2eq)/ IT Equipment Energy $(\mathrm{kWh})$

For data centers with electricity as the only energy source this is mathematically equivalent to multiplying the PUE by the data center's carbon emission factor (CEF). The CEF is determined by the $\mathrm{Co}_{2}$ intensity of grid electricity $\left(\mathrm{kgCo}_{2} \mathrm{eq} / \mathrm{kWh}\right)$ in a given region.

$$
C U E=\sum_{i} C E F_{\mathrm{i}} \times E_{i}
$$

The ideal CUE value is 0 , a level that indicates that no carbon emissions are associated with a data center's operations.

The Table 1 shows the most common CEF of electric resources. It can be discovered that the CEF of fossil fuels is the largest, like coal and petroleum, while the CEF of green resources is relatively small, like wind energy and solar energy.

Table 1 The most commonly used energy carbon emission factor [8-10]

\begin{tabular}{cc}
\hline Energy & Carbon emission factor $/\left(\mathrm{gCO}_{2} \mathrm{e} \cdot \mathrm{kWh}^{-1}\right)$ \\
\hline Nuclear & 15 \\
Coal & 968 \\
Petroleum & 440 \\
Oil & 890 \\
Water & 13.5 \\
wind energy & 29.0 \\
solar energy & 53.0 \\
\hline
\end{tabular}

The Table 2 comparing to expenditure and the CEF, the third party Power. Purchase Agreements, PPAs and Renewable Energy Certificates, RECs as is less than power grid and DG. As a result, the unit cost of PPAs and REC of green power is less and the CEF is 0.

Table 2 units of energy costs and carbon emissions factor [9]

\begin{tabular}{ccc}
\hline Energy & $\begin{array}{c}\text { The unit cost } /(\$ . \\
\left.\mathrm{kWh}^{-1}\right)\end{array}$ & Carbon emission factor $/\left(\mathrm{gCO}_{2} \mathrm{e} \cdot \mathrm{kWh}^{-1}\right)$ \\
\hline Power grid & 5.0 & 586 \\
PPA & 6.0 & 0 \\
REC & 0.5 & 0 \\
DG & 30.0 & 1056 \\
\hline
\end{tabular}

Data Center Energy Productivity (DCeP). The DCeP can be defined as the ratio of the useful work produced by the date center and the total energy consumption to produce that work. The DCeP is calculated as follows:

$$
\mathrm{DCeP}=\frac{\text { Userful work produced }}{\text { Total energy consumed to produce that work }}
$$

In terms of effective load, green grid organization is designing a sort of systems which are organized by 8 productivity representatives to appraisal $\boldsymbol{D C e p}$.It includes weighing the utility of $\boldsymbol{C P U}$, the work load efficiency of operation system, transmitted byte per kilowatt per hour, etc. 


\section{Measurement Model for Energy Efficiency in Data Centers}

In assessing energy efficiency opportunities, it is important to carefully measure component and system power consumption by using monitoring equipment as well as computer models.

Energy efficiency measurement model of data center is mainly composed of the energy consumption data center $P_{\text {total }}$, IT equipment energy consumption $P_{\mathrm{IT}}$, and IT equipment efficiency $\eta$ measurement. Measurement model for energy efficiency in data centers is shown in Fig. 2.

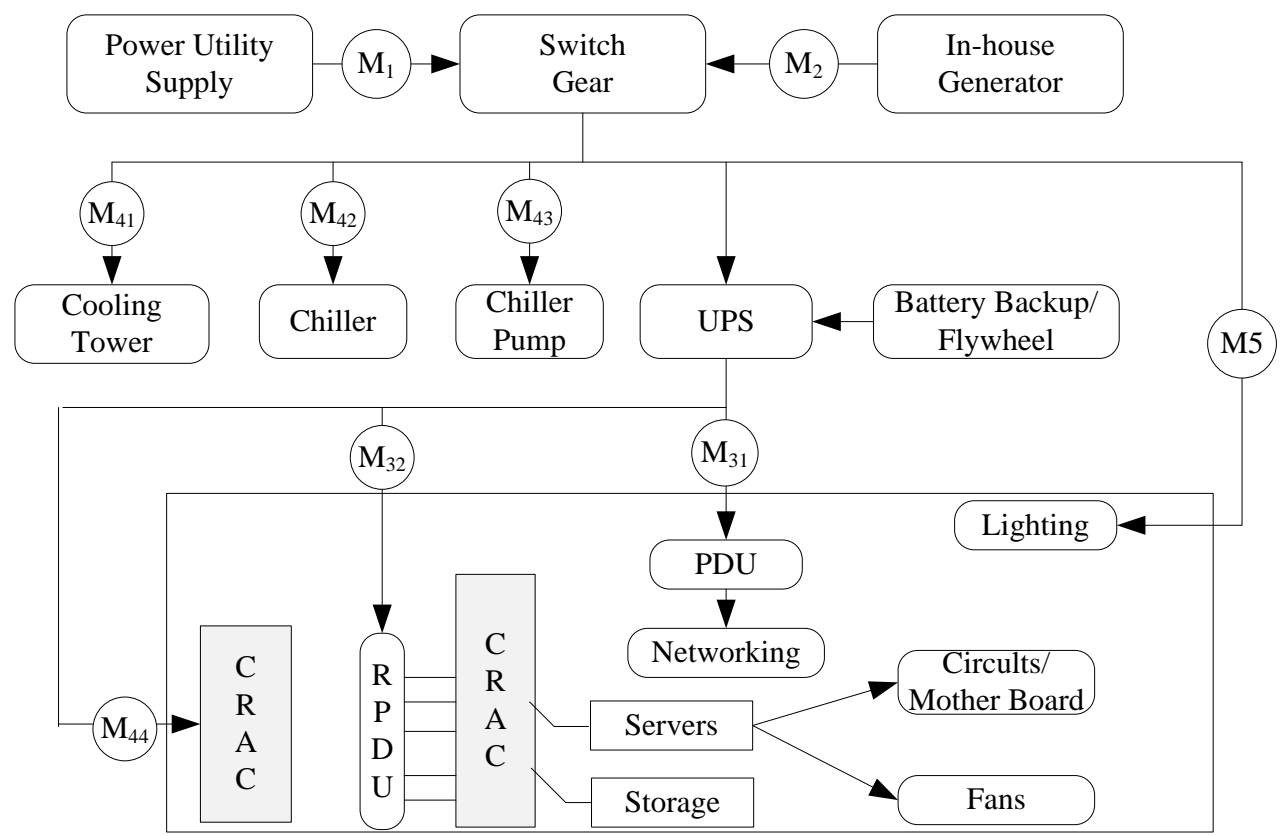

Figure 2. Measurement Model for Energy Efficiency in Data Centers

(1). The Energy Consumption $\boldsymbol{P}_{\text {total }}$ In normal working condition, the electricity of data center is provided by power utility supply, and the measuring point should be chosen before power utility supply input into the transformers, which is the $\mathrm{M}_{1}$ in Fig. 2 . When there is breakdown of the power utility supply, the electrical power of the In-house generator, which is the $\mathbf{M}_{2}$ in Fig. 2, serves as the total energy consumption measuring point.

(2). IT Equipment Energy Consumption $\boldsymbol{P}_{\text {IT. }}$ IT equipment energy consumption should be the energy consumption $P_{\text {total }}$, and it is increase measuring workload and costs in larger quantity of IT equipment. Therefore, in practice, measurement point is UPS of output or PDUs of input, which is $\mathrm{M}_{3}$ in Fig. 2. measure value sum that IT equipment power consumption $P_{\mathrm{IT}}$.

(3). Measuring of IT Equipment Efficiency. $\eta$. There is a set of real time tasks denoted as $T=\left\{T_{1}, T_{2}, \ldots, T_{\mathrm{n}}\right\}$, IT equipment data processing task size denoted as $L(T)=\left\{L T_{1}, L T_{2}, \ldots, L T_{\mathrm{n}}\right\}$ and IT equipment energy consumption denoted as $\boldsymbol{P}_{\mathrm{IT}}(\boldsymbol{T})=\left\{P_{\mathrm{IT}}\left(T_{1}\right), P_{\mathrm{IT}}\left(T_{2}\right), \ldots, P_{\mathrm{IT}}\left(T_{\mathrm{n}}\right)\right\}$.

It can be expressed mathematically as:

$$
\eta(T)=\frac{L(T)}{P_{\mathrm{IT}}(T)}, P_{\mathrm{IT}}(T) \neq 0
$$

For each computing nodes $i(1 \leq i \leq N)$, where computing nodes has $\mathrm{N}$ nodes. CPU frequency in $t$ time denoted as $f_{i}(t)$, CPU usage frequency in $t$ time denoted as $\omega_{i}(t)$ and CPU consumption power denoted as $p_{i}(t)$.

$L_{i}(t)$ and $P_{I T(i)}(t)$ can be expressed as:

$$
L_{i}(t)=f_{i}(t) \omega_{i}(t), \quad P_{\mathrm{IT}(i)}(t)=p_{i}(t)
$$

Sum on $\mathrm{N}$ nodes in between 0 and $T$, energy efficiency $\eta(T)$ as shown below: 


$$
\eta(T)=\frac{L(T)}{P_{\mathrm{IT}}(T)}=\frac{\sum_{i=1}^{N} \int_{0}^{T} L_{i}(t) d t}{\sum_{i=1}^{N} \int_{0}^{T} P_{\mathrm{IT}(i)}(t) d t}=\frac{\sum_{i=1}^{N} \int_{0}^{T} f_{i}(t) \omega_{i}(t) d t}{\sum_{i=1}^{N} \int_{0}^{T} p_{i}(t) d t}
$$

\section{Summary}

This paper has shown the need for the industry to develop standards and metrics for measuring energy efficiency in data centers. Such metrics will be vital tools for mangers to use when assessing the performance of their facilities and determining where resources should be focused to create improvement.

As the environment continues to be affected by data center emission, governments will start to regulate the energy use and force data centers to make improvements. By taking initiative and creating metrics the industry can be prepared to demonstrate progress toward energy efficiency.

\section{Acknowledgement}

This research was supported by the national natural science foundation of China (Grant No. 61540055), the doctor`s scientific research foundation of Hezhou University(No. HZUBS201506), and the project of Guangxi university of science and technology research (No.2013YB242).

\section{References}

[1] U.S. Energy information admin. annual energy review 2011 [R]. DOE/EIA-0384(2011). Washington D. C., 2011: 3-4.

[2] Greenpeace "likes" facebook's New Datacenter, But Wants a Greener Friendship [Online]. Available: http://www.greenpeace.org/internation/en/press/releases/Greenpease-likes-Face-boo books-new-datacentre-but wants -a-greener-friendship.

[3] J.Koomey. Growth in Data Center Electricity Use: 2005 to 2010[Online]. Available: http://www.analyticspress.com/datacenters.html.

[4] Belady C. Green Grid Data Center Power Efficiency Metrics: PUE and DCiE [R/OL]. http://www.THEGREE-NGRID.org, 2008.

[5] Data center industry leaders reach agreement on guiding principles for energy efficiency metrics [EB/OL]. (2010-02-01) [2013-07-01]. http:www.energystar.gov/ ia/parners/prod_development/ downloads/DataCenters_AgreementGuidingPrinciples.pdf.

[6] Belady C. Green Grid Data Center Power Efficiency Metrics: PUE and DCiE [R/OL]. http://www.THEGREE-NGRID.org, 2008.

[7] Belady C, Azecedo D, Patterson M, Pouchet J, Tipley R. Carbon Usage Effectiveness (CUE): A green grid data center sustainability metric[R]. The Green Grid. Technical Report, December 2010.

[8] Gao P X, Curtis A P, Wong B, et al. It's not easy being green[C]. Proceedings of the ACM SIGCOMM 2012, Helsinki, Finland, 2012: 211-222.

[9] Ren Chuan-gang, Wang Di, Urgaokar B, et al. Carbon-aware energy capacity planning for datacenters [C]. Proceedings of the IEEE International of Computer and Telecommunication Systems (MASCOTS 2012). Washington, USA, 2012: 391-400.

[10] Oseph B H, Spadaro V, Langlois L. Greenhouse gas emissions of electricity generation chains: Assessing the difference [J]. IAEA Bulletin, 2000, 42(2): 19-28. 